\title{
Código de Gobierno Societario en la Argentina: análisis del nivel de cumplimiento
}

\section{Corporate Governance Code in Argentina: analysis of the level of compliance}

Mg. Eliana Barco es profesora del Departamento de Ciencias de la Administración de la Universidad Nacional del Sur (UNS) (ebarco@uns.edu.ar) (https://orcid.org/0000-0003-0462-8308)

Dra. Anahí Briozzo es profesora del Departamento de Ciencias de la Administración de la Universidad Nacional del Sur (UNS) e investigadora del Instituto de Investigaciones Económicas y Sociales del Sur (IIESS, UNS-CONICET) (abriozzo@uns.edu.ar) (https://orcid.org/0000-0002-7865-2821)

\section{Resumen}

Un Código de Gobierno Corporativo es un conjunto de normas, principios y recomendaciones de comportamiento y funcionamiento, considerados como las mejores prácticas para el buen gobierno de las organizaciones para mejorar la transparencia, divulgación y rendición de cuentas. En el presente trabajo se analiza el grado de cumplimiento del Código de Gobierno Societario en la Argentina, que es de presentación obligatoria para las empresas que hacen oferta pública de sus títulos. El trabajo empírico se lleva cabo en una muestra de 20 empresas de capitales nacionales, excluyendo al sector financiero y a las compañías que realizan cross listing de sus valores. Se determina el nivel de cumplimiento del código presentado por cada firma desde dos perspectivas: la autoevaluación realizada por la propia emisora, según el principio de cumplir o explicar, y el análisis efectuado por un evaluador externo, a partir de la información públicamente disponible. Los resultados muestran que el nivel de cumplimiento es mayor desde la óptica de la autoevaluación, y para las empresas de mayor tamaño. Los principios de mayor cumplimiento, independientemente del tamaño de la compañía, son los referidos a partes relacionadas y a la función de auditoría. En las firmas de mayor capitalización también resulta elevada la adhesión al principio de ética empresarial. El principio referido a remunerar de manera justa y responsable presenta el menor cumplimiento en todas las empresas estudiadas.

\begin{abstract}
A Corporate Governance Code is a set of rules, principles, and recommendations for behavior and operation, considered as the best practices for the good governance of organizations, in order to improve transparency, disclosure, and accountability. This paper analyzes the degree of compliance with the Corporate Governance Code in Argentina, where the Code is mandatory for companies that make a public offering of their securities. The empirical work is carried out in a sample of 20 national capital companies, excluding the financial sector and the companies that cross-list their values. The analysis of the level of compliance of the code presented by each firm is developed from two perspectives: the self-assessment carried out by the company, according to the principle of comply or explain, and the analysis performed by an external evaluator, based on publicly available information. The results show that the level of compliance is higher from the perspective of self-assessment and for larger companies. The principles of greater compliance, regardless of the size of the company, are those related to related parties and the audit function. In companies with greater capitalization, adherence to the principle of business ethics is also high. The principle referred to remunerate in a fairly and responsibly way presents the lowest compliance in all the studied companies.
\end{abstract}

\section{Palabras clave I keywords}

Código de gobierno corporativo, principio cumplir o explicar, Argentina, gobernanza, rendición de cuentas, accionistas, buenas prácticas.

Corporate Governance Code, comply or explain principle, Argentina, governance, accountability, shareholders, good practices.

Cómo citar: Barco, E., y Briozzo, A. (2020). Código de Gobierno Societario en la Argentina: análisis del nivel de cumplimiento. Retos Revista de Ciencias de la Administración y Economía, 10(19), 45-63. https://doi.org/10.17163/ret.n19.2020.03 


\section{Introducción y estado de la cuestión}

Los escándalos corporativos ocurridos a principios de siglo en diferentes países pusieron sobre la agenda el tema de Gobierno Corporativo (GC), empujando a los diferentes actores a buscar mejores prácticas de gobernanza. Como consecuencia, se inició un proceso de regulación y legislación tendiente a prevenir los fraudes financieros y corrupción administrativa, aumentar la veracidad de la información, generar credibilidad y proteger a stakeholders en general. Cabe destacar que dentro de los objetivos para el desarrollo sostenible planteados por la Organización de las Naciones Unidas se encuentra el de "Promover sociedades, justas, pacíficas e inclusivas" (Objetivo 16). En particular, una de las metas que se plantea se vincula directamente con el GC, al indicar que se busca "Crear a todos los niveles instituciones eficaces y transparentes que rindan cuentas" (meta 16.6) (ONU, 2015).

El objetivo de la presente investigación es realizar un análisis y medición del Gobierno Corporativo en la Argentina, a través del estudio del Código de Gobierno Societario (CGS) presentado por las empresas. Se define un índice cuantitativo del grado de cumplimiento del CGS a partir de un análisis exhaustivo de los informes presentados por las empresas participantes de los mercados de valores de la Argentina y sujetas a la reglamentación de la Res. 606/12 de la Comisión Nacional de Valores (CNV), que se basa en el principio de cumplir o explicar. Dicho principio está presente en la mayoría de los códigos de GC del mundo (Seidl, Sanderson \& Roberts 2013). Fue propuesto originariamente por el Comité Cadbury en el Reino Unido (Committee on the Financial Aspects of Corporate Governance, 1992), e implica que las empresas pueden cumplir con los requerimientos del código, o explicar por qué no lo hacen, en contraste con los regímenes obligatorios como la Ley Sarbanes-Oxley de los Estados Unidos.

El nivel del cumplimiento del CGS se estudia desde dos puntos de vista: el declarado por la empresa, por un lado, y el que surge del análisis documental de las explicaciones brindadas en el CGS, por el otro. Posteriormente, se verifica si existen diferencias en el cumplimiento en función del tamaño de la empresa, y se describe cuáles son los principios con mayor y menor adhesión y dispersión en las evaluaciones. La población bajo estudio son las 42 emisoras de acciones de capital nacional de los mercados de valores argentinos, cuyos títulos no se encuentren listados en otros mercados, excluido el sector financiero que se encuentra sujeto a normativa diferencial. La muestra consiste en 20 empresas, tomándose las diez de mayor capitalización bursátil y las diez de menor capitalización.

\subsection{Códigos de Gobierno Corporativo: el principio de cumplir o explicar}

Un Código de Gobierno Corporativo es un conjunto de normas, de principios y recomendaciones de comportamiento y funcionamiento considerados como las mejores prácticas para el buen gobierno de las organizaciones para mejorar la transparencia, divulgación y rendiciones de cuentas de directivos (Zattoni \& Cuomo, 2008; Fornero, 2007). Los códigos están basados en los conceptos doctrinarios inherentes a las mejores prácticas en la materia vigentes en un momento para regular las relaciones entre los interesados. 
La presencia de códigos se vuelve relevante cuando otros mecanismos, como el sistema legal, las políticas y normas de la empresa, los sistemas de control, entre otros, no son efectivos. En ausencia de otros mecanismos de protección para los accionistas, se diseñan códigos que buscan mejorar el funcionamiento del consejo y la calidad de la información y rendición de cuentas (Fornero, 2007).

Zattoni y Cuomo (2008) adhieren a este enfoque, señalando que los códigos pretenden suplir las deficiencias de los sistemas de gobernanza, regulando los aspectos que generan mayores riesgos para los accionistas y son fuente de conflicto con los directivos.

Los códigos se han desarrollado y evolucionado antes que las leyes propias de la materia. Son emitidos y legitimados por el mismo Estado o por bolsas de valores, y se instrumentan por medio de leyes societarias, regulaciones o estructuras de cumplimento privadas.

Fornero (2007) indica que existe cierta convergencia a nivel mundial sobre los contenidos y recomendaciones de los códigos sobre transparencia, rendición de cuentas, independencia y consejo de administración. El énfasis en algunos temas se adapta al ambiente económico de cada país y sus principales problemas de GC. Remarca también que la existencia de un código es más relevante en los países con sistema legal débil. Un estudio de Zattoni y Cuomo (2008) reveló que países common law son más propensos a desarrollar códigos que países civil law, quienes adoptan los códigos frente al riesgo de perder legitimación más que por razones de eficiencia o voluntad de mejorar sus prácticas.

Gran parte de los códigos adoptan el principio cumplir o explicar: la organización no está obligada a cumplir con los postulados enunciados en los códigos de Buenas Prácticas, pero en caso de no hacerlo, debe explicar por qué no lo hace. El principio cumplir o explicar adopta un enfoque flexible, considerando que no existe una única forma o medida para todas las organizaciones, y que, bajo ciertas circunstancias, las organizaciones pueden no cumplir o no aplicar para algunas recomendaciones. La esencia del mismo es permitir justificar el no cumplimiento para casos particulares o situaciones especiales (Seidl, Sanderson \& Roberts 2012).

La efectividad de las iniciativas de autorregulación ha sido debatida en la literatura, con resultados mixtos. Con respecto al no cumplimiento, Merkl-Davies y Brennan (2007) indican que el silencio puede ser una táctica particular en relación con las divulgaciones de ley blanda (soft law), donde hay poca o ninguna supervisión por los reguladores.

Considerando los costos de implementación de las buenas prácticas de gobernanza, el nivel cumplimiento del código de GC puede vincularse conceptualmente con el tamaño de la empresa, aunque la evidencia empírica no es uniforme. Campbell et al. (2009) estudian las empresas polacas y encuentran que el nivel de cumplimiento no tiene relación estadística con el tamaño de la empresa. El 16\% de las empresas cumplen el 100\% del código. El principio que reúne el mayor incumplimiento es la independencia de los miembros del consejo (74\%), seguido por la ausencia de comités de auditoría y remuneraciones (66\%).

Benavides Franco y Mongrut Montalvan (2010) encuentran que entre el 2001 (fecha de la primera introducción del requerimiento del código de GC en Colombia) hasta el 2006, 101 empresas habían emitido su código de GC, de las cuales 43 perte- 
necían al sector no-financiero. El nivel de cumplimiento promedio, medido como el porcentaje de respuestas positivas en relación al total, fue de $46 \%$ en ese periodo, con una correlación positiva muy baja con el tamaño de la empresa. El nivel de cumplimiento del código ha ido en aumento en las empresas colombianas, llegando al 71\% en el periodo 2008-2014 (Lagos Cortés, Betancourt Ramírez \& Gómez Betancourt, 2018). El mayor cumplimiento se da en las recomendaciones de la dimensión referida a la asamblea de accionistas (81\%), mientras que la menor adhesión se presenta en las recomendaciones para resolución de controversias $(60 \%)$.

En las empresas chilenas, el grado de adopción de las prácticas de gobierno corporativo es más baja que en el caso colombiano, rondando en el año 2015 el 40\% para las de mayor liquidez bursátil, y 30\% para el resto (Moraga \& Ropero, 2018). Para ambos grupos de empresas, el grupo de principios con mayor cumplimiento fueron los referidos a la gestión y control de riesgos. La tendencia en el cumplimiento del código ha sido levemente positiva en el periodo 2015-2017, incrementándose en un promedio de 6\% (Torres, Troncoso \& Ramírez, 2019).

Briano-Turrent y Poletti-Hughes (2017) construyen un índice de GC para las firmas de los principales índices bursátiles de Argentina, Brasil, Chile y México, y encuentran que en el periodo 2004-2010 el valor promedio del índice fue de 64\%, con una fuerte relación positiva con el tamaño de la empresa. Por lo tanto, considerando los antecedentes conceptuales y empíricos, se propone la siguiente hipótesis:

H1: El cumplimiento del CGS tiene una relación inversa con el tamaño de la empresa.

Bianchi et al. (2011) encuentran que el 85.9\% de las compañías italianas cumplen formalmente con las recomendaciones del principio de transacciones con partes relacionadas, pero solo el 32,6\% han implementado esas recomendaciones de una manera que los autores consideran satisfactoria. Esta brecha es mayor para las firmas no financieras y las de menor tamaño.

Luo y Salterio (2014) estudian la adopción de las buenas prácticas en Canadá y señalan que solo el 7\% las adopta por completo. El cumplimiento por explicación en lugar de por adopción se aplica a las prácticas que tienen altos costos para la empresa. Para aquellas firmas muy cercanas al cumplimiento completo, los principios que no se verifican son mayormente aquellos relacionados con la independencia del consejo y sus subcomités (independencia del presidente del consejo, búsqueda y reuniones de directores independientes y total independencia de los comités de remuneración y nominación).

Shrives y Brennan (2017) analizan 100 empresas del Reino Unido en dos momentos del tiempo con cambio en la normativa (2004/05 y 2011/12). Encuentran que el 43\% de las empresas en el 2011/12, y el 63\% en el periodo anterior, no cumplen con alguno de los principios. El análisis muestra un incremento de las estrategias retóricas empleadas en la justificación del no cumplimiento, con una preponderancia de explicaciones engañosas más que de razonamientos convincentes y significativos. Teniendo en cuenta los antecedentes presentados, se formula la siguiente hipótesis:

H2: La autoevaluación de la empresa difiere de los resultados de una evaluación de un analista externo 


\subsection{Marco normativo del GC en la Argentina}

La Argentina posee un mercado de capitales de escaso desarrollo como otros países de la región y de los mercados emergentes. Este mercado se caracteriza por concentración de la propiedad, la escasa liquidez y bajo nivel de actividad de los inversores institucionales e intermediarios y bajo nivel de transparencia. En este contexto, los incentivos de las empresas para la adhesión voluntaria de buenas prácticas y códigos de GC son escasos. Esto hace que la mayor adhesión esté dada por el componente Institucional $^{1}$ fundamentalmente (CEF, 2005).

En el aspecto público, se observaba que la Argentina poseía menor performance que otros países de la región en cuanto a GC institucional y requerimientos formales. Por lo tanto, existe margen para mejorar el GC institucional, la efectiva aplicación de acciones sancionatorias y fortalecer la seguridad jurídica y la protección del derecho de los accionistas minoritarios (Villegas, 2006).

El marco regulatorio vigente en la Argentina para empresas bajo régimen de oferta pública comprende la Ley General de Sociedades (Ley 19550), la Ley de Mercado de Capitales (Ley 26831) y las Normas CNV (2013). Este conjunto normativo establece la obligación de contar con un comité de auditoría, integrado como mínimo por tres miembros del directorio (consejo de administración) y cuya mayoría deberá ser independiente. Se establecen también los criterios de independencia de directores y obligación de presentar declaraciones juradas respecto a la condición de independencia. Asimismo, los auditores externos deben ser independientes, se definen las partes relacionadas y se establece la obligatoriedad de informar actos y contratos y aprobación previa cuando sea necesario.

Con respecto al CGS, en el 2006 la CNV emitió la Resolución General 493/06 por la cual las empresas que cotizan títulos valores en la bolsa debían contestar un cuestionario de 14 preguntas, relacionadas con el cumplimiento de los principios que sustentan al Gobierno Corporativo. Posteriormente, se emitió la Resolución 516/07(CNV), que aprueba el CGS propio de las sociedades autorizadas para ofertar públicamente las acciones representativas de su capital social. Esta resolución establece la obligatoriedad de presentar de un informe sobre cumplimiento de GC en oportunidad de prepararse los estados contables de ejercicio de la entidad, en forma independiente de estos, para ser difundidos con la misma modalidad de un hecho relevante. La aplicación del código es discrecional y voluntaria, complementada con la metodología de cumplir o explicar mediante un informe a ser preparado por el órgano de administración y a ser incluido como integrante de la memoria social. En el año 2012 la CNV reemplazó el informe anual a presentar sobre Gobierno Corporativo por uno más completo, según lo establecido en la Res. CNV 606/12. Esta Resolución crea de un Código Gobierno Societario, estructurado a partir de principios y recomendaciones, con requisitos y contenidos mínimos que deben cumplir las

1 La dimensión institucional, también conocida como externa, es normativa. Está representada por el marco regulatorio, el sistema jurídico y la red de instituciones de un determinado país. Está definido por las leyes y regulaciones vigentes en cada país por lo que es de aplicación obligatorio. 
entidades autorizadas a efectuar oferta pública de sus valores negociables ${ }^{2}$. Los nueve principios se resumen en la Tabla 1.

Tabla 1. Contenidos del CGS (Res. CNV 606/12)

\begin{tabular}{|c|c|c|c|}
\hline Principio & Título & N.R & Contenido \\
\hline 1 & $\begin{array}{l}\text { Transparentar la rela- } \\
\text { ción entre la emisora, } \\
\text { el grupo económico que } \\
\text { encabeza y/o integra y } \\
\text { sus partes relacionadas }\end{array}$ & 3 & $\begin{array}{l}\text { Existencia de i) normativa de autorización de } \\
\text { transacciones entre partes relacionadas, ii) me- } \\
\text { canismos de prevención de conflictos de inte- } \\
\text { rés; iii) mecanismos para prevenir uso indebi- } \\
\text { do de información privilegiada. }\end{array}$ \\
\hline 2 & $\begin{array}{l}\text { Sentar las bases para } \\
\text { una sólida administra- } \\
\text { ción y supervisión de la } \\
\text { emisora }\end{array}$ & 7 & $\begin{array}{l}\text { Rol del órgano de administración (OA) en la } \\
\text { planificación estratégica, política de GC y RSE, } \\
\text { selección, evaluación, remuneración y sucesión } \\
\text { de gerentes de primera línea; control de gestión } \\
\text { y de desempeño de la primer línea gerencial; } \\
\text { participación de miembros independientes en } \\
\text { el OA (mínimo 20\%); existencia y funciona- } \\
\text { miento del comité de nombramientos (comité } \\
\text { no obligatorio); selección, capacitación, eva- } \\
\text { luación y sucesión de los miembros del OA y } \\
\text { límites para su participación en otras organi- } \\
\text { zaciones. }\end{array}$ \\
\hline 3 & $\begin{array}{l}\text { Avalar una efectiva po- } \\
\text { lítica de identificación, } \\
\text { medición, administra- } \\
\text { ción y divulgación del } \\
\text { riesgo empresarial }\end{array}$ & 1 & $\begin{array}{l}\text { Existencia de políticas de gestión integral de } \\
\text { riesgos actualizadas, de un comité de gestión } \\
\text { de riesgos, indicar la metodología empleada } \\
\text { (COSO, ISO 31000, etc). }\end{array}$ \\
\hline 4 & $\begin{array}{l}\text { Salvaguardar la integri- } \\
\text { dad de la información } \\
\text { financiera con auditorías } \\
\text { independientes }\end{array}$ & 1 & $\begin{array}{l}\text { Mayoría independiente en el Comité de Audito- } \\
\text { ría (comité obligatorio), existencia de función } \\
\text { de auditoría interna; análisis de la independen- } \\
\text { cia de los auditores externos, política de rota- } \\
\text { ción de los auditores externos. }\end{array}$ \\
\hline 5 & $\begin{array}{l}\text { Respetar los derechos de } \\
\text { los accionistas }\end{array}$ & 6 & $\begin{array}{l}\text { Existencia de reuniones informativas periódi- } \\
\text { cas con los accionistas; mecanismos de infor- } \\
\text { mación a inversores y sitio web; mecanismos } \\
\text { de promoción de la participación de accionistas } \\
\text { minoritarios e institucionales; igualdad entre } \\
\text { acción y voto; mecanismos de protección fren- } \\
\text { te a tomas de control; dispersión accionaria de } \\
\text { min. } 20 \% \text {; política de dividendos transparente. }\end{array}$ \\
\hline
\end{tabular}

2 La Res. CNV 797/19 modifica el código de gobierno societario, entrando en vigencia a partir del 31 de diciembre de 2019. 


\begin{tabular}{|c|l|c|l|}
\hline 6 & $\begin{array}{l}\text { Mantener un vínculo di- } \\
\text { recto y responsable con } \\
\text { la comunidad }\end{array}$ & 1 & $\begin{array}{l}\text { Existencia de sitio i) web de acceso público } \\
\text { con información relevante y canal de comuni- } \\
\text { cación; ii) Balance de responsabilidad social y } \\
\text { ambiental con verificación de auditor externo } \\
\text { independiente; iii) adopción de normas (GRI, } \\
\text { ISO 26000, etc.). }\end{array}$ \\
\hline 7 & $\begin{array}{l}\text { Remunerar de forma jus- } \\
\text { ta y responsable }\end{array}$ & 1 & $\begin{array}{l}\text { Existencia de comité de remuneraciones, com- } \\
\text { posición y funcionamiento; o detallar cumpli- } \\
\text { miento de estas funciones dentro del OA. }\end{array}$ \\
\hline 8 & $\begin{array}{l}\text { Fomentar la ética empre- } \\
\text { sarial }\end{array}$ & 1 & $\begin{array}{l}\text { Existencia de i) código de conducta empresaria } \\
\text { y aplicación a OA, empleados, clientes, provee- } \\
\text { dores; ii) mecanismos para recibir denuncias } \\
\text { en forma confidencial: iii) políticas para la ges- } \\
\text { tión y resolución de denuncias }\end{array}$ \\
\hline 9 & $\begin{array}{l}\text { Profundizar el alcance } \\
\text { del código }\end{array}$ & 1 & $\begin{array}{l}\text { Inclusión de las previsiones del CGS en el Es- } \\
\text { tatuto social }\end{array}$ \\
\hline
\end{tabular}

Nota: N.R se refiere al número de recomendaciones incluidas en el principio.

Fuente: Elaboración propia

\subsection{Análisis y medición del GC en la Argentina}

La evolución del concepto Gobierno Corporativo y la trascendencia que el mismo ha adquirido en los últimos años, ha llevado al desarrollo de índices que permitan evaluar y medir las buenas prácticas de Gobierno Corporativo de las empresas. Existe una gran cantidad de índices, tanto comerciales como académicos, e institucionales.

La Bolsa de Valores de Argentina no ha desarrollado aun un índice institucional de Gobierno Corporativo. Los antecedentes de medición se detallan a continuación. En el año 2006, el CEF diseñó el primer índice de GC para Argentina: el Índice de transparencia y difusión (ITD), cuyo objetivo fue aportar una medida cuantitativa y objetiva que permitiera realizar un seguimiento de la evolución del Gobierno Corporativo de las empresas en Argentina. El ITD está basado en la información pública (proveniente de diversas fuentes) de cada empresa. Bebczuk (2005) construyó un índice de GC para empresas cotizantes en la Bolsa de Valores. Se basa en un índice de transparencia y difusión, el cual se construye con información pública; y en un índice de Gobierno que se completa con la información de encuestas a empresas. La tasa de respuesta de estas encuestas ha sido muy baja. Por lo tanto, el índice de transparencia es considerado la medida de GC, dada la alta correlación con el mismo. Al momento de la construcción, la adopción del código era voluntaria, por lo cual la desventaja del mismo es que solo mostraba la información que la empresa quería mostrar públicamente; generalmente la misma se refiere a aspectos contables.

IDEA (2010) realizó un análisis de la RG 516/07 de la CNV en 30 compañías. El objetivo del estudio era analizar el cumplimiento real (adhesión proactiva) vs el formal (adhesión reactiva). Gutiérrez y Marcos (2018) analizan una muestra de 14 
empresas de distintos sectores, ${ }^{3}$ incluidas empresas del sector financiero y durante el período del año 2013 al 2016, encontrando que en la mayoría de las empresas analizadas el cumplimiento (auto-evaluado) del CGS supera el 70\%.

\section{Materiales y métodos}

\subsection{Diseño, población y muestra}

El enfoque de investigación empleado es mixto de alcance descriptivo, utilizando herramientas cualitativas para el análisis documental de los CGS presentados por las emisoras y un posterior análisis cuantitativo de los resultados.

El diseño de la investigación es no experimental, ya que "sólo se observan los fenómenos en su ambiente natural para después analizarlos" (Hernández Sampieri et al., 2010, p. 149). En cuanto al alcance temporal, el análisis se ha realizado a los CGS presentados en los ejercicios finalizados en 2015/2016. En este trabajo se analizan fuentes de datos secundarias, en particular el CGS presentado por las empresas en el periodo 2015/2016. La información se encuentra disponible en forma libre y gratuita en el sitio web de la CNV.

La población objeto de estudio son las empresas de capital abierto participantes emisoras de acciones en los mercados de valores argentinos, listadas en el panel general, que cumplen con ciertos requisitos. Partiendo de un total de 97 compañías listadas, se procede a excluir: a) aquellas que se encuentran listadas en mercados internacionales y las reguladas por el Banco Central de la República Argentina (por estar sujetas a normativas diferenciales de gobierno corporativo), y b) aquellas que integran el panel PYME, aun cuando por su capitalización pudieran estar incluidas como empresas a analizar, por no estar obligadas a presentar el CGS. Este procedimiento de exclusión obedece a evaluar compañías que presenten información bajo el mismo régimen, eliminando así a las emisoras que presentan variaciones no por su comportamiento, política o decisión, sino por encontrarse alcanzadas por un marco regulatorio diferente respecto al GC. De esta forma, se eliminan las variaciones institucionales y se analizan únicamente organizaciones comparables entre sí en cuanto a la legislación y forma de presentación de la información. Por lo tanto, la población se reduce a 42 firmas, la muestra seleccionada en este estudio consiste un total de 20 empresas: las diez compañías de mayor y las diez de menor capitalización bursátil en la fecha indicada.

\subsection{Herramientas de análisis}

El análisis de los datos se realiza en diversas etapas: una primera etapa de análisis cualitativo de datos y luego una etapa cuantitativa de síntesis de resultados que permite arribar a un índice que resume el nivel del cumplimiento de CGS.

En primer lugar, se lleva a cabo un análisis documental del CGS de cada empresa estudiada en función del grado de cumplimiento declarado por la misma.

3 Este trabajo incluye empresas del sector financiero y que realizan cross listing de sus títulos. Entre el trabajo de Gutiérrez y Marcos (2018) y la presente investigación coinciden solo tres empresas estudiadas. 
Se asigna un valor de 0 si la empresa declara no cumplir la recomendación; un valor de 0,5 si declara cumplimiento parcial y un valor de 1 si declara cumplimiento total. Para cada uno de los nueve principios que integran el CGS se determina el cumplimiento relativo según el siguiente ratio:

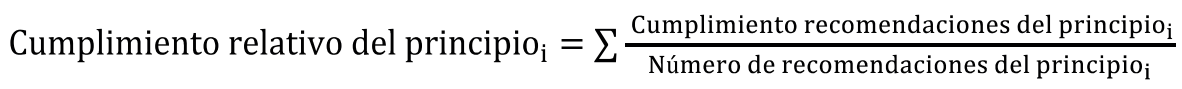

Por ejemplo, el principio I está integrado por las siguientes recomendaciones. Si una empresa declara cumplimiento total en la recomendación I.1 (1 punto), parcial en la recomendación I.2 (0,5 puntos) y no cumplir la recomendación I.3 (0 puntos), el ratio de cumplimiento relativo sería:

$$
\text { Cumplimiento relativo del } \text { principio }_{\mathrm{I}}=\sum \frac{1+0,5+0}{3}=50 \%
$$

Con esta información declarada se realiza un ordenamiento de los principios en función de su nivel de cumplimiento relativo, a fines de determinar los principios de mayor y menor cumplimiento declarado, desagregado por tamaño y comparando ambos grupos.

Posteriormente se realiza un nuevo análisis del CGS, a fines de estudiar la consistencia entre la autoevaluación propuesta y la justificación brindada, desde el punto de vista de un analista externo que accede sólo a la información públicamente disponible. Para este análisis se utiliza la Revisión de textos, comparando los requerimientos especificados en los principios de CGS con las respuestas de las empresas. El grado de cumplimiento se determina considerando: i) si la respuesta dada por la empresa es completa y se ajusta a las recomendaciones del CGS, ii) si se detallan los procesos, políticas, acciones tal como la recomendación lo solicita, y iii) en los casos que aplica, la contrastación entre las respuestas y la información pública disponible, externa al CGS. Por ejemplo, al analizar la independencia del directorio, la rotación de los auditores, la cantidad de comités, la existencia de página web, se tiene en cuenta no sólo la respuesta de la emisora en su CGS sino también la información pública disponible sobre la misma y se valida la respuesta dada con la evidencia externa.

En forma similar a la etapa anterior, se calcula para cada principio su nivel de cumplimiento relativo. Esta nueva clasificación es comparada con la obtenida por autoevaluación, a fines de determinar los principios con mayor distancia entre lo declarado por la empresa y la opinión de un analista externo, a través de la diferencia absoluta entre ambas calificaciones. La dispersión dentro de un mismo principio se mide en términos del desvío estándar.

La siguiente etapa consiste en la elaboración de un índice cuantitativo que resuma el nivel del cumplimiento del CGS para cada empresa. Se elabora un índice lineal, donde los nueve principios tienen el mismo peso. Se elige la metodología lineal para construir el índice por interpretar la motivación de la Res. CNV 606/12, de establecer nueve principios con distintas recomendaciones, como el interés del órgano de contralor de brindar igual importancia a cada principio de gobierno corporativo. La construcción del índice se realiza según el siguiente ratio: 
Índice de cumplimiento del CGS $=\frac{\sum_{i=1}^{9} \text { Cumplimiento relativo del principio } i}{9}$

De esta forma, si una empresa posee un nivel de cumplimiento relativo de $50 \%$ en los principios I a V, de 100\% en los principios VI a VII y de $0 \%$ en el principio IX, su índice de cumplimiento del CGS será:

Índice de cumplimiento del CGS $=\frac{0,5+0,5+0,5+0,5+0,5+1+1+1+0}{9}=61,1 \%$

Este cálculo se realiza considerando el cumplimiento de cada principio tanto en función de la autoevaluación de cada empresa como del análisis de un analista externo.

Las diferencias en la distribución del cumplimiento de los principios y del índice general según el tamaño de la empresa, así como entre la autoevaluación y la visión del analista externo, se evalúan mediante el test de U Mann-Whitney (también conocido como prueba de suma de rangos Wilcoxon), que es una prueba no paramétrica aplicada a dos muestras independientes.

\section{Resultados}

En la primera sección se muestran los resultados de la autoevaluación que realizan las empresas, y del grado de cumplimento auto-asignado, obteniendo un ranking de cumplimientos de criterios. La siguiente sección estudia el cumplimiento de los criterios desde la perspectiva de un evaluador externo, mientras que la sección 3.3 presenta la comparación de ambos enfoques.

\subsection{Autoevaluación del informe sobre el grado de cumplimiento del Có- digo de Gobierno Societario}

Como puede observarse en la Figura 1, la autoevaluación no presenta grandes diferencias entre empresas grandes y pequeñas con relación al ranking de cumplimiento de principios. En ambos grupos, el principio de mayor cumplimiento es el Principio I (Transparentar la relación entre la emisora y sus partes relacionadas) y el de menor es el VII (Remunerar en forma justa). Los principios VI (Mantener vínculo responsable con la comunidad) y VIII (Fomentar la ética empresarial) son los que presentan mayores diferencias en el grado de cumplimiento según el tamaño de empresa.

La Tabla 2 presenta los valores medios según el tamaño, error estándar y p-value del test de Mann Whitney. El grado de cumplimiento auto-asignado es estadísticamente mayor para las empresas de mayor tamaño al considerar el índice general, como se indica en la hipótesis H1. Sin embargo, al estudiar el cumplimiento por principio, se observa que esta diferencia es relevante sólo para los principios VI (Mantener un vínculo directo y responsable con la comunidad) y VIII (Fomentar la ética empresarial), por lo que las diferencias en el nivel de adhesión al CGS no son homogéneas entre los principios. 


\section{Figura 1. Autoevaluación de los principios del cumplimiento del CGS según tamaño de la empresa}

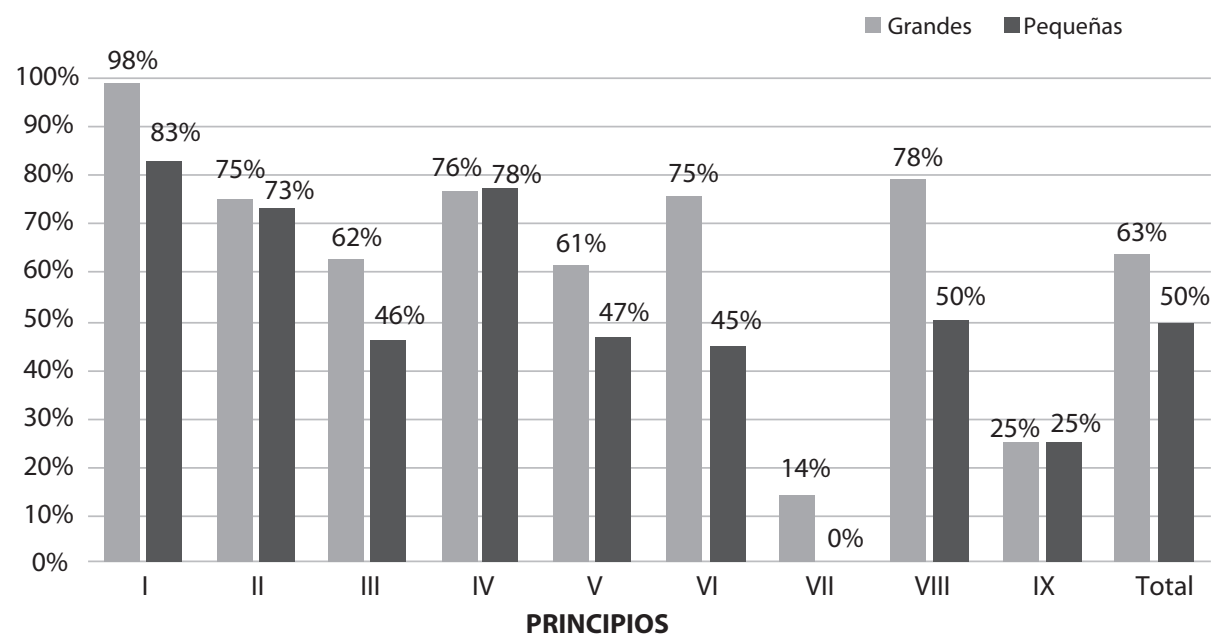

Fuente: Elaboración propia

Tabla 2. Autoevaluación de los principios del cumplimiento del CGS según tamaño de la empresa

\begin{tabular}{|l|c|c|c|c|c|}
\hline \multirow{2}{*}{ Principio } & \multicolumn{2}{|c}{ Pequeñas } & \multicolumn{2}{c|}{ Grandes } & \multirow{2}{*}{ p-value } \\
\cline { 2 - 5 } & Media & $\begin{array}{c}\text { Desví } \\
\text { Estándar }\end{array}$ & Media & $\begin{array}{c}\text { Desvío } \\
\text { Estándar }\end{array}$ & \\
\hline P1E & $83.4 \%$ & $35.0 \%$ & $98.3 \%$ & $5.4 \%$ & 0.465 \\
\hline P2E & $73.4 \%$ & $9.3 \%$ & $74.8 \%$ & $11.1 \%$ & 0.762 \\
\hline P3E & $51.1 \%$ & $20.3 \%$ & $62.0 \%$ & $29.4 \%$ & 0.387 \\
\hline P4E & $77.8 \%$ & $19.3 \%$ & $76.6 \%$ & $13.7 \%$ & 0.667 \\
\hline P5E & $51.9 \%$ & $11.7 \%$ & $61.1 \%$ & $9.7 \%$ & 0.125 \\
\hline P6E & $45.0 \%$ & $19.7 \%$ & $75.0 \%$ & $26.4 \%$ & $0.016^{* *}$ \\
\hline P7E & $0.0 \%$ & $0.0 \%$ & $14.2 \%$ & $30.1 \%$ & 0.147 \\
\hline P8E & $49.8 \%$ & $36.0 \%$ & $78.3 \%$ & $35.2 \%$ & $0.051 *$ \\
\hline P9E & $25.0 \%$ & $26.4 \%$ & $25.0 \%$ & $26.4 \%$ & 1.000 \\
\hline Total-E & $49.8 \%$ & $12.6 \%$ & $62.7 \%$ & $8.3 \%$ & $0.021 * *$ \\
\hline
\end{tabular}

Nota: P1E denota que se analiza el principio 1 según la auto-evaluación de la empresa. Se presentan los p-values del test de Mann-Whitney. Significatividad estadística al 5\% (**) y al 10\% (*).

Fuente: Elaboración propia 
La dispersión en el cumplimiento de los principios también presenta diferencias en función del tamaño de la empresa. Mientras que los principios con mayor dispersión entre grandes compañías son el VIII (Fomentar ética empresarial), VII (Remunerar en forma justa y responsable y III (Administración de riesgos), en las firmas de menor tamaño son los principios VIII (Fomentar ética empresarial), I (Grupo económico y partes relacionadas) y IX (Profundizar el alcance del código).

\subsection{Evaluación externa del informe sobre el grado de cumplimiento del Código de Gobierno Societario}

En la Figura 2 se observa el cumplimiento de la evaluación externa por principio, y segregado por tamaño de la empresa. Se observan disparidades y diferencias entre ambos grupos. Mientras que en las empresas grandes los principios de mayor cumplimiento son el I (Transparentar la relación entre la emisora, el grupo económico que encabeza y/o integra y sus partes relacionadas), VI (Mantener un vínculo directo y responsable con la comunidad) y VIII (Fomentar la ética empresarial), en las pequeñas se cumple mayormente con los principios I, II (Sentar las bases para una sólida administración y supervisión de la emisora) y IV (Salvaguardar la integridad de la información financiera con auditorías independientes). El principio con menor cumplimiento es el IX (Profundizar el alcance del código en las firmas grandes) y el VII (Remunerar de forma justa y responsable) en las pequeñas.

\section{Figura 2. Evaluación externa de los principios del cumplimiento del CGS según tamaño de la empresa}

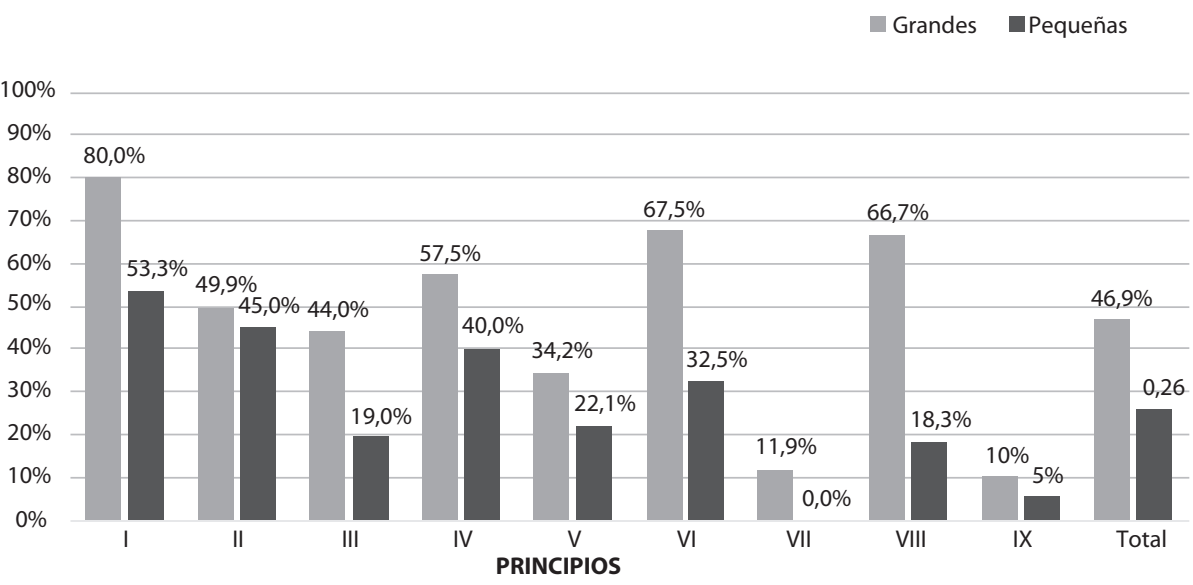

Fuente: Elaboración propia.

La Tabla 3 permite apreciar que la disparidad en el cumplimiento de los principios entre empresas grandes y pequeñas es mayor al considerar la evaluación de un analista externo. Si bien se observa nuevamente una diferencia significativa a nivel 
general de cumplimiento, el comportamiento por principio difiere notablemente del observado en la Tabla 2. Únicamente el principio II (Sentar las bases para una sólida administración y supervisión de la emisora) y el principio IX (Profundizar el alcance del código) presentan cumplimientos similares entre ambos grupos.

\section{Tabla 3. Evaluación externa de los principios del cumplimiento del CGS según tamaño de la empresa}

\begin{tabular}{|l|c|c|c|c|c|}
\hline \multirow{2}{*}{ Principio } & \multicolumn{2}{|c|}{ Pequeñas } & \multicolumn{2}{c|}{ Grandes } & \multirow{2}{*}{ p-value } \\
\cline { 2 - 6 } & Media & $\begin{array}{c}\text { Desví } \\
\text { Estándar }\end{array}$ & Media & $\begin{array}{c}\text { Desví } \\
\text { Estándar }\end{array}$ & \\
\hline P1A & $53.4 \%$ & $34.0 \%$ & $79.9 \%$ & $18.9 \%$ & $0.076^{*}$ \\
\hline P2A & $45.2 \%$ & $8.0 \%$ & $49.9 \%$ & $9.4 \%$ & 0.250 \\
\hline P3A & $19.0 \%$ & $13.7 \%$ & $44.0 \%$ & $28.4 \%$ & $0.047^{* *}$ \\
\hline P4A & $40.2 \%$ & $24.3 \%$ & $57.6 \%$ & $15.8 \%$ & $0.093^{*}$ \\
\hline P5A & $22.0 \%$ & $9.2 \%$ & $34.3 \%$ & $9.5 \%$ & $0.012^{* *}$ \\
\hline P6A & $32.5 \%$ & $20.6 \%$ & $67.5 \%$ & $23.7 \%$ & $0.004^{* * *}$ \\
\hline P7A & $0.0 \%$ & $0.0 \%$ & $12.0 \%$ & $25.3 \%$ & 0.147 \\
\hline P8A & $18.4 \%$ & $35.6 \%$ & $66.7 \%$ & $35.0 \%$ & $0.011^{* *}$ \\
\hline P9A & $5.0 \%$ & $15.8 \%$ & $10.0 \%$ & $21.1 \%$ & 0.542 \\
\hline Total-A & $26.10 \%$ & $8.6 \%$ & $46.80 \%$ & $9.8 \%$ & $0.001^{* * *}$ \\
\hline
\end{tabular}

Nota: P1A denota que se analiza el principio 1 según la evaluación del analista externo. Se presentan los p-values del test de Mann-Whitney. Significatividad estadística al $1 \%(* * *), 5 \%(* *)$ y al $10 \%(*)$. Fuente: Elaboración propia.

La dispersión en el cumplimiento de principios bajo la perspectiva del analista externo también difiere según el tamaño de empresa. Mientras que en las pequeñas compañías los puntos de mayor dispersión son el VIII (Fomentar la ética empresarial), I (Grupo económico y partes relacionadas) y IV (Auditorías independientes), se observa que en las grandes son el VIII (Fomentar la ética empresarial), III (Administración del riesgo) y VII (Remunerar de forma justa y responsable). Es interesante mencionar que, para las empresas de mayor tamaño, los tres principios con mayor dispersión desde esta óptica son los mismos que los observados bajo la autoevaluación de la empresa. Sin embargo, en las firmas pequeñas, el tercer principio con mayor dispersión presenta un cambio: es el IX (Profundizar el alcance del código) bajo la autoevaluación, y el IV (Auditorías independientes) desde la óptica del analista externo.

En síntesis, los resultados obtenidos, tanto bajo la autoevaluación de la empresa como bajo un analista externo, muestran que existe evidencia a favor de la hipótesis H1: el cumplimiento del CGS tiene una relación inversa con el tamaño de la empresa. 


\subsection{Análisis comparativo de evaluaciones}

En la Figura 3 se observa la comparación del cumplimiento auto-asignado y el cumplimiento de la evaluación externa por principio, y segregado por tamaño de la empresa. Se observa también el grado de cumplimiento general.

\section{Figura 3. Comparación de evaluaciones del cumplimiento del CGS según tamaño de la empresa}

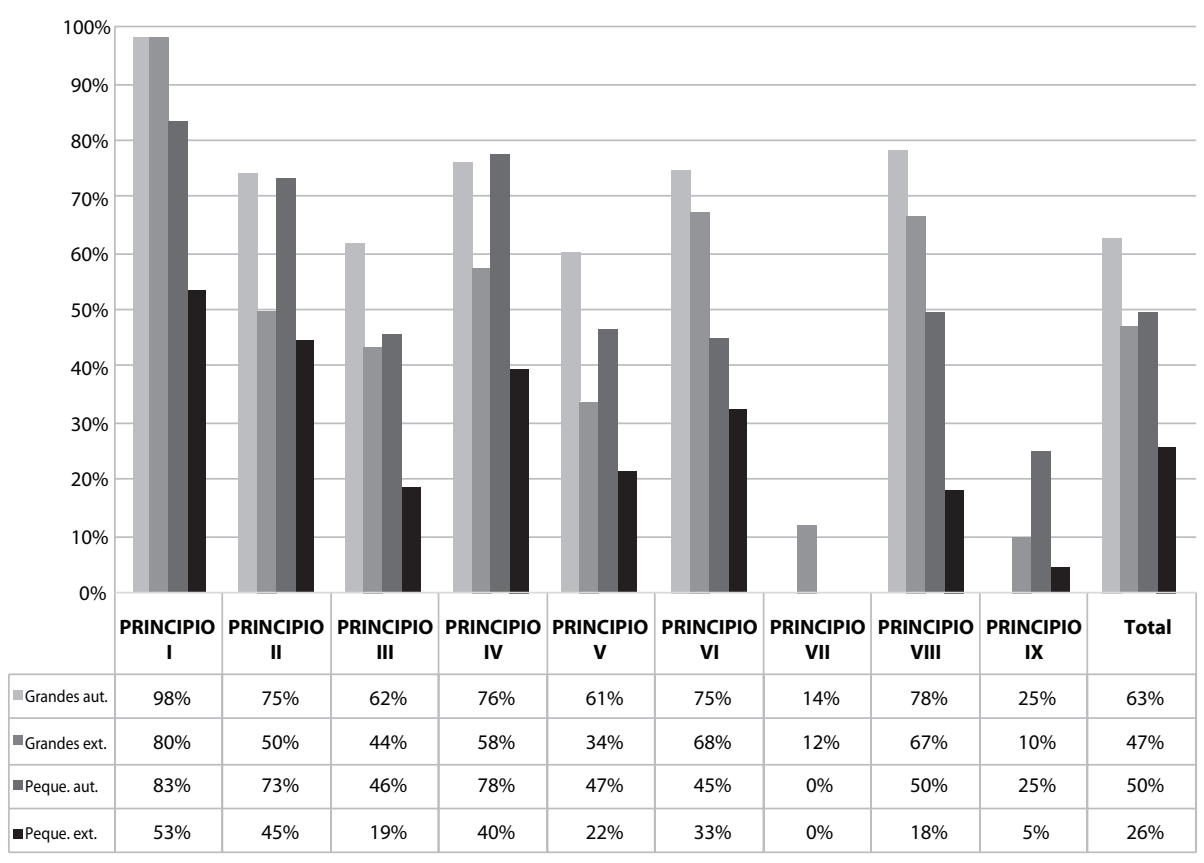

Fuente: Elaboración propia.

La Tabla 4 presenta el cumplimiento por principio para toda la muestra, desde dos perspectivas; autoevaluación y analista externo. Como puede observarse, las distribuciones son diferentes para todos los principios excepto en el VI (Mantener un vínculo directo y responsable con la comunidad) y VII (Remunerar de forma justa y responsable). De esta forma, se encuentra evidencia a favor de H2: La autoevaluación de la empresa difiere de los resultados de una evaluación de un analista externo. 


\section{Tabla 4. Evaluación de los principios del cumplimiento del CGS según la empresa y el analista externo}

\begin{tabular}{|l|c|c|c|c|c|}
\hline \multirow{2}{*}{ Principio } & \multicolumn{2}{|c|}{ Autoevaluación } & \multicolumn{2}{c|}{ Analista externo } & \multirow{2}{*}{ P-value } \\
\cline { 2 - 6 } & Media & Des. Estand & Media & Des. Estand & \\
\hline P1 & $90.9 \%$ & $25.5 \%$ & $66.7 \%$ & $30.0 \%$ & $0.0008 * * *$ \\
\hline P2 & $74.1 \%$ & $10.0 \%$ & $47.6 \%$ & $8.8 \%$ & $0.0000 * * *$ \\
\hline P3 & $56.8 \%$ & $25.4 \%$ & $31.5 \%$ & $25.2 \%$ & $0.0036 * * *$ \\
\hline P4 & $77.2 \%$ & $16.3 \%$ & $48.9 \%$ & $21.9 \%$ & $0.0001 * * *$ \\
\hline P5 & $56.7 \%$ & $11.4 \%$ & $28.2 \%$ & $11.1 \%$ & $0.0000^{* * *}$ \\
\hline P6 & $60.0 \%$ & $27.4 \%$ & $50.0 \%$ & $28.1 \%$ & 0.3218 \\
\hline P7 & $7.1 \%$ & $21.9 \%$ & $6.0 \%$ & $18.5 \%$ & 0.9172 \\
\hline P8 & $64.1 \%$ & $37.6 \%$ & $42.6 \%$ & $42.4 \%$ & $0.0910 *$ \\
\hline P9 & $25.0 \%$ & $25.6 \%$ & $7.5 \%$ & $18.3 \%$ & $0.0196 * *$ \\
\hline Total & $56.3 \%$ & $12.3 \%$ & $36.5 \%$ & $13.9 \%$ & $0.0001 * * *$ \\
\hline
\end{tabular}

Nota: Se presentan los p-values del test de Mann-Whitney. Significatividad estadística al 1\% (***), 5\% $(* *)$ y al $10 \%(*)$.

Fuente: Elaboración propia.

A continuación, se analizan las características de los principios, según su grado de adhesión y según su dispersión entre evaluaciones.

\subsubsection{Principios de mayor adhesión}

Al evaluar los principios y su nivel de cumplimiento, se observa que el principio de mayor cumplimiento, tanto en la evaluación de la empresa como en la evaluación externa, para todas las empresas es el PRINCIPIO I: Transparentar la relación entre la emisora, el grupo económico que encabeza y/o integra y sus partes relacionadas.

En las empresas grandes, es un principio con alto grado de cumplimiento y concordancia entre la evaluación de las emisoras y la evaluación del analista externo, con un $100 \%$ de cumplimiento en dos de sus tres recomendaciones. En las empresas pequeñas el cumplimiento asignado es solo del 53\%, inferior al $83 \%$ indicado por las propias emisoras en sus análisis. Las principales diferencias obedecen a que las emisoras consideran cumplidas las recomendaciones por el hecho de acatar la ley, y no tienen acciones complementarias más allá de la normativa vigente.

Únicamente en la evaluación del analista externo, el tamaño de la organización contribuye como elemento diferenciador en el cumplimiento del principio: las empresas de mayor tamaño tienen mejores evaluaciones propias como externas, debido a que las empresas grandes además de las recomendaciones legales toman acciones complementarias para transparentar las relaciones.

En las empresas grandes, el PRINCIPIO VIII: Fomentar la ética empresarial tiene muy buenas evaluaciones, ubicándose en segundo lugar, con menor diferencia 
entre ambas evaluaciones (11\% neto). En general, esta diferencia obedece a la falta de explicaciones sobre los procesos y mecanismos para la gestión de denuncias.

En las empresas de menor tamaño se observa menor auto-cumplimiento (cuarto principio en el orden de auto-cumplimiento), y una mayor diferencia en cuanto a la evaluación externa, que arroja un cumplimiento del 18\% del principio, con siete emisoras que no cumplen el mismo, a pesar de que cinco de ellas se autoevalúan con algún grado de cumplimiento. Las principales diferencias obedecen a que las emisoras informan cumplimiento aun cuando reconocen no poseer mecanismos o procesos, o indicar que no consideran necesario implementarlos.

En comparación con el grupo de grandes empresas, se puede afirmar que el tamaño es un factor de diferenciación (Tablas 2 y 3): las empresas grandes cuentan con Códigos de conducta, con más mecanismos y procesos, así como con tercerización de los mismos; mientras en las empresas menores carecen de ellos y no tienen reconocida la necesidad, muchas veces justificado en el tamaño de la misma.

El PRINCIPIO IV (Salvaguardar la integridad de la información financiera con auditorías independientes,) posee similar evaluación propia en ambas muestras, ubicándose en tercer lugar para las grandes empresas y segundo para las pequeñas. Dentro de este principio, la recomendación con mayor cumplimiento para todas las empresas es la evaluación anual por parte del comité de auditoría de los auditores externos. La recomendación con menor cumplimiento se refiere a la rotación de los miembros de la comisión fiscalizadora y del auditor externo para todas las empresas, junto con la existencia de una función de auditoría interna para las de menor tamaño.

\subsubsection{Principios de menor adhesión}

En el extremo opuesto, los principios de menor cumplimiento son, para ambas muestras:

- PRINCIPIO VII (Remunerar de forma justa y responsable): el cumplimiento del principio es muy bajo, no existiendo diferencias entre la evaluación realizada por el analista externo y la autoevaluación de la misma empresa. En particular, este principio trata la existencia del comité de remuneraciones y sus funciones. Es el único criterio donde ambas evaluaciones no presentan dispersión. El tamaño de la emisora no es un factor de diferenciación, ya que todas las empresas de menor tamaño se autoevalúan con incumplimiento, coincidiendo con la evaluación externa.

- PRINCIPIO IX (Profundizar el alcance del código): en ambos grupos de empresas es un principio de bajo cumplimiento, donde las empresas manifiestan cumplir parcialmente solo por el hecho de presentar el informe de cumplimiento del Cogido de Gobierno Societario establecido por la Resolución CNV 606/12. Adicionalmente indican que no analizan incluir cuestiones de gobernanza en su Estatuto social, dado que consideran que la legislación es suficiente. La evaluación externa es aún menor, presentado mayor dispersión absoluta las empresas pequeñas. Si se mide la variación porcentual, las diferencias entre ambas evaluaciones es importante, en especial en las pequeñas: es el principio de mayor variación porcentual. La comparación entre ambos grupos de empresas no permite afirmar que existan diferencias en función del tamaño de la organización (Tablas 2 y 3 ). 


\section{Conclusiones y discusión}

El objetivo general de este trabajo es analizar el CGS de las empresas participantes de los mercados de valores argentinos y elaborar un índice cuantitativo del cumplimiento del mismo, evaluando diferencias en función del tamaño de la empresa y del tipo de evaluación. Los resultados obtenidos muestran que existen diferencias entre el índice autoasignado por la propia emisora y el índice asignado por la evaluación del analista externo. Es interesante mencionar que, si bien el cumplimiento general del CGS difiere según el tamaño de las empresas, al analizarlo por principio se observa que esta diferencia no es homogénea entre principios.

Un objetivo secundario es determinar cuáles son los aspectos de mayor y menor cumplimiento. Los principios referidos a Partes Relacionadas (Principio I) y a la función de Auditoría (Principio IV) son las de mayor cumplimiento sin distinción por tamaño. Esto tiene fundamento en el cumplimiento de disposiciones legales que llevan a cumplir también el Código. En las empresas grandes el principio de Ética Empresarial (Principio VIII) tiene alto grado de cumplimiento.

Los principios de mayor cumplimiento corroboran la dimensión institucional del GC, así como lo que Fornero (2007) denomina noción centrada en los accionistas: el GC como una visión restringida, basada en el control por medio de mecanismos normativos: leyes, códigos de conducta, regulaciones, código de buenas prácticas. Los principios de menor cumplimiento son los referentes al Comité de Remuneraciones (resultado coincidente con Campbell et al., 2009) y a la Política de GS y su inclusión en estatuto.

Se concluye que la opinión de un analista externo no coincide con autoevaluación de las emisoras, la cual es superior a la evaluación externa. Esto indica que el analista externo realiza un análisis más exigente, resultado similar al obtenido por Bianchi et al. (2011). En coincidencia con Merkl-Davies y Brenann (2007), se observa en ocasiones que la emisora se autoevalúa como cumplimiento total aun cuando no cumple ni presenta explicaciones. Esto podría obedecer a que la misma empresa conoce los procesos, políticas y/o motivos que sustentan su autoevaluación. No sucede lo mismo con un tercero (parte interesada, inversor, organismo de control) que analiza el informe: carece de información interna para comprender y evaluar. Estos resultados señalan la utilidad de la contratación voluntaria de un auditor externo del CGS, que pueda interactuar con la empresa y certificar la información presentada, como ocurre en la Bolsa de Lima. De esta forma, aumentaría la confianza en la información presentada en el CGS y colaboraría con la protección de todos los grupos interesados.

El estudio cuenta con las siguientes limitaciones: evaluación realizada solo con fuentes de información secundaria, y reducido número de empresas que participan del mercado de capitales. Como líneas de investigación futuras, se puede ampliar la muestra incorporando más emisoras, contactar a las empresas para que brinden más información y feedback sobre cuestiones no explicadas; analizar información pública de la empresa que responda puntos nos indicados en el informe y contactar inversores para conocer su percepción del CGS.

Los resultados de este trabajo presentan una medición del Gobierno Corporativo en la Argentina, que implica una contribución significativa al análisis integral de los informes de Gobierno en Latinoamérica. De esta forma, esta investigación se inserta 
en la línea de trabajos que analizan cómo los directivos de las empresas aplican los códigos de CG, lo cual permite estudiar la efectividad del sistema cumplir o explicar. Por otra parte, los estudios locales sobre gobierno corporativo permiten a los organismos de control del mercado de capitales (en la Argentina la Comisión Nacional de Valores) diseñar mecanismos que fomenten la transparencia y la rendición de cuentas, en línea con los objetivos para el desarrollo sostenible de la ONU (2015).

\section{Apoyos y soporte financiero de la investigación}

Entidad: Universidad Nacional del Sur (UNS)

País: Argentina

Ciudad: Bahía Blanca

Proyecto subvencionado: Desafíos del gobierno corporativo en empresas argentinas: su relación con la rentabilidad, financiamiento y riesgo

Código de proyecto: 24/C049.

\section{Referencias}

Bebczuk, R. N. (2005). Corporate Governance and Ownership: Measurement and Impact on Corporate Performance and Dividend Policies in Argentina. Inter-American Development Bank - Latin American Research Network - Research Network Working paper \#R-516.

Benavides-Franco, J., \& Mongrut-Montalvan, S. (2010). Governance codes: facts or fictions? a study of governance codes in Colombia. Estudios Gerenciales, 26(117), 85-102.

Bianchi, M., Ciavarella, A., Novembre, V., \& Signoretti, R. (2011). Comply or explain: Investor protection through the Italian corporate governance code. Journal of Applied Corporate Finance, 23(1), 107-121.

Briano-Turrent, G., \& Poletti-Hughes, J. (2017). Corporate governance compliance of family and non-family listed firms in emerging markets: Evidence from Latin America. Journal of Family Business Strategy, 8(4), 237-247.

Campbell, K., Jerzemowska, M., \& Najman, K. (2009). Corporate governance challenges in Poland: Evidence from "comply or explain" disclosures. Corporate Governance: The international journal of business in society, 9(5), 623-634.

CEF (2005). El Gobierno Corporativo en Argentina. Nota de Política $N^{\circ} 5$. Centro de Estabilidad Financiera. Recuperado de: https://bit.ly/37Ufm0X [Fecha de consulta 24 de febrero de 2014].

CEF (2006). El Gobierno Corporativo de los Bancos en Argentina. Nota de Política $\mathrm{N}^{\circ}$ 6. Centro de Estabilidad Financiera. Recuperado de: https://bit.ly/394wmC3 [Fecha de consulta 11 de marzo de 2014].

Comisión Nacional de Valores (2007). Resolución General 516/2007. Código de Gobierno Societario. Buenos Aires, 11 de octubre de 2007. Recuperado de: https://bit.ly/3b8pqFu [Fecha de consulta 23 de febrero de 2014].

Comisión Nacional de Valores (2012). Resolución General 606/2012. Modificación Normas CNV (N.T. 2001) - Código de Gobierno Societario. Buenos Aires, 23 de mayo de 2012. Recuperado de: https://bit.ly/2Un9dpS [Fecha de consulta 4 de marzo de 2014].

Comisión Nacional de Valores (2013). Texto Ordenado Normas Comisión Nacional de Valores 2013. Aprobadas por Ley 26.831 y Decreto Reglamentario 1023/2013. Recuperado de: https://bit.ly/396rOdQ [Fecha de consulta 15 de marzo de 2014]

Comisión Nacional de Valores (2019). Resolución General 797/19. Recuperado de: https://bit. ly/391zdLC [Fecha de consulta 10 de agosto de 2019]. 
Committee on the Financial Aspects of Corporate Governance (1992). Report with Code of Best Practice [Cadbury Report]. London: Gee Publishing. Recuperado de: https://bit.ly/36NcFNe [Fecha de consulta 20 de febrero de 2014].

Decreto 677/01 (2001). Poder Ejecutivo Nacional. Régimen de transparencia de la oferta pública. Buenos Aires, 22 de mayo de 2001. Recuperado de: https://bit.ly/2UslF85 [Fecha de consulta 11 de noviembre de 2013].

Fornero, R. (2007). El valor de parecer bueno. Perspectiva financiera de las buenas prácticas de gobernanza empresaria. XXVII Jornadas de Administración Financiera. Recuperado de: https://bit.ly/2S9JJtu [Fecha de consulta 22 de julio de 2013].

Gutiérrez, O., \& Marcos, D. (2018). Gobierno Corporativo: Medición en empresas cotizantes en la Argentina. 38 Jornadas de Docentes en Administración Financiera (SADAF). Recuperado de https://bit.ly/2u4aYxH [Fecha de consulta 15 de marzo de 2019].

Hernández Sampieri, R., Collado Fernández, C., \& Baptista Lucio, P. (2014). Metodología de la investigación, $6 .^{\mathrm{a}} \mathrm{Ed}$. México DF: McGraw-Hill.

IDEA (2010). Principios de Buen Gobierno. Recuperado de: https://bit.ly/2RT0R7H [Fecha de consulta 18 de abril de 2014].

Lagos Cortés, D., Betancourt Ramírez, J., \& Gómez Betancourt, G. (2018). Relación entre gobierno corporativo, control familiar y desempeño en empresas colombianas. Innovar, 28(69), 85-98.

Ley No 19550. Ley General de Sociedades. Modif. Ley no 26.994/27.077. Boletín Oficial de la Nación Argentina, Buenos Aires, Argentina, 8 de octubre de 2014. Recuperado de: https:// bit.ly/2u6MvYF [Fecha de consulta 15 de marzo de 2019].

Ley N ${ }^{\circ}$ 26831. Mercado de Capitales. Boletín Oficial de la Nación Argentina, Buenos Aires, Argentina, 27 de diciembre de 2012. Recuperado de: https://bit.ly/2RRN8OG [Fecha de consulta 18 de abril de 2014].

Luo, Y., \& Salterio, S. E. (2014). Governance quality in a "comply or explain" governance disclosure regime. Corporate Governance: An International Review, 22(6), 460-481.

Merkl-Davies, D., \& Brennan, N (2007). Discretionary Disclosure Strategies in Corporate Narratives: Incremental Information or Impression Management? Journal of Accounting Literature, 26, 116-196

Moraga, H., \& Ropero, E. (2018). Gobierno Corporativo y desempeño financiero de las empresas más importantes del mercado bursátil chileno. Revista Venezolana de Gerencia, 23(81), 145-162.

ONU (2015). Objetivos de desarrollo sostenible. Recuperado de: https://bit.ly/2GPBspd [Fecha de consulta 15 de noviembre de 2019].

Shrives, P. J., \& Brennan, N. M. (2017). Explanations for corporate governance non-compliance: A rhetorical analysis. Critical Perspectives on Accounting, 49, 31-56.

Seidl, D., Sanderson, P., \& Roberts, J. (2013). Applying the 'comply-or-explain' principle: discursive legitimacy tactics with regard to codes of corporate governance. Journal of Management Gove, 17(3), 791-826.

Torres, F. E. A., Troncoso, R. C., \& Ramírez, V. S. (2019). Adopción de prácticas de gobierno corporativo del mercado chileno en el trienio 2015-2017. Visión de Futuro, 23(2), 1-17.

Villegas, M. (2006). Gobierno Corporativo en Argentina. Univ. Del CEMA. Recuperado de: https:// bit.ly/37WfHQr [Fecha de consulta 12 de abril de 2014].

Zattoni, A., \& Cuomo, F. (2008). Why Adopt Codes of Good Governance? A Comparison of Institutional and Efficiency Perspectives. Corporate Governance: An International Review, 16(1), 1-15. 